\title{
The effect of etomidate or propofol on brainstem function during anesthesia induction: a bispectral index-guided study
}

This article was published in the following Dove Press journal:

Drug Design, Development and Therapy

\section{Huibao Zheng* \\ Yuexin Zhu* \\ Kaizheng Chen \\ Xia Shen}

Department of Anesthesiology, The Eye, Ear, Nose and Throat Hospital of Fudan University, Shanghai Medical College of Fudan University, Shanghai, People's Republic of China

*These authors contributed equally to this work
Correspondence: Xia Shen

Department of Anesthesiology, The Eye, Ear, Nose and Throat Hospital, Fudan University, 83 Fenyang Road, Shanghai 20003I, People's Republic of China

Tel +862164377134

Fax +862164373416

Email zlsx@yahoo.com
Purpose: To compare the effect of etomidate versus propofol infusion on hemodynamic profiles, spontaneous breathing, and corneal reflex during the induction of anesthesia.

Methods: Adult patients $(n=80)$ were randomized to receive etomidate (Group E, $n=40$ ) or propofol (Group $\mathrm{P}, \mathrm{n}=40$ ) infusion during anesthesia induction. Throughout induction, mean blood pressure and heart rate were monitored. Time to loss of consciousness (LOC), bispectral index (BIS), existence of spontaneous breathing, and corneal reflex at LOC were recorded.

Results: Fewer changes in hemodynamic profile occurred in Group E compared with Group $\mathrm{P}$ during induction of anesthesia. The mean time to LOC was shorter with etomidate than propofol (129.5 s vs $189.5 \mathrm{~s}, P<0.0001)$. BIS was lower in Group E compared with Group $\mathrm{P}$ at LOC (46.3 vs 52.9, $P=0.0141$ ). More patients exhibited spontaneous breathing in Group E compared with Group P at LOC $(80 \%$ vs $17.5 \%, P<0.0001)$. Similarly, more patients maintained corneal reflex in Group E compared with Group P (34 patients vs 4 patients, $P<0.0001)$. The incidence of etomidate-induced myoclonus was $17.5 \%$.

Conclusion: Compared with propofol infusion, etomidate infusion during anesthesia induction had fewer effects on the hemodynamic profile of patients. Among patients who received etomidate, the BIS value was lower at LOC, and more patients displayed spontaneous breathing and corneal reflex.

Trial Registry Number: China Clinical Research Information Service, ChiCTR-IOR $-17011050$

Keywords: anesthesia, etomidate, propofol, consciousness monitors, bispectral index

\section{Background}

Propofol and etomidate are commonly used to induce anesthesia. Both agents exhibit rapid initiation and brief duration of action. However, etomidate is safer for elderly and vulnerable patients who are hemodynamically unstable. ${ }^{1,2}$ The mechanism through which etomidate promotes hemodynamic stability may be related to the baroreceptor function and a lack of effect on the sympathetic nervous system. ${ }^{3,4}$ By contrast, Goodchild et al reported that clinical concentrations of propofol did not cause negative inotropic effects or relax arteries. ${ }^{5}$

In addition to direct effects on the myocardium and peripheral vasculature, additional underlying mechanisms through which anesthetics cause cardiovascular effects include central nervous system depression. ${ }^{3-6}$ However, even at the same level of anesthesia guided by the bispectral index (BIS) value, Möller et al reported 
that etomidate caused fewer hemodynamic changes than propofol during the induction of anesthesia. ${ }^{7}$

BIS is a measure of activity in the cortex and not the brainstem. The cardiovascular tone was generated in the brainstem. Therefore, etomidate may have fewer effects on the brainstem compared with propofol. If this is the case, then brainstem function, reflected by hemodynamic profiles, respiratory function, and corneal reflex, should be less affected by etomidate. In the current study, the primary endpoint was hemodynamic changes with either etomidate or propofol infusion during induction of anesthesia. The secondary endpoints were BIS, presence of spontaneous breathing, and corneal reflex at loss of consciousness (LOC).

\section{Methods}

We registered the current study with the China Clinical Research Information Service (ChiCTR-IOR-17011050, approval date: 2017/04/04) and followed the principles of the Declaration of Helsinki. The Hospital Ethics Committee (Shanghai EYE \& ENT Hospital) approved the study, and patients provided written informed consent. Eighty patients with American Society of Anesthesiologists physical status I or II and who were undergoing functional endoscopic sinus surgery were enrolled. Exclusion criteria included adrenocortical insufficiency, allergies to the study agents, or declining to participate.

No medication was administered in advance. In the operating room, BIS (BIS ${ }^{\mathrm{TM}} \mathrm{XP}$ sensor), $\mathrm{ECG}$, heart rate (HR), noninvasive blood pressure, and peripheral oxygen saturation were monitored (Mindray BeneView T8, Shenzhen Mindray Bio-Medical Electronics Co., Ltd). A peripheral 20-gauge venous cannula was inserted into the forearm to facilitate infusion of $0.9 \%$ normal saline at a rate of $400 \mathrm{~mL} / \mathrm{h}$. Oxygen was delivered via facemask at a rate of $10 \mathrm{~L} / \mathrm{min}$ once induction of anesthesia was initiated. First, patients received $3 \mathrm{~mL}$ of $2 \%$ lidocaine. Etomidate $(2 \mathrm{mg} / \mathrm{mL}$, Jiangsu Nhwa Pharmaceutical, Xuzhou, China) or propofol (10 mg/mL, Fresenius Kabi Pharmaceutical Co. Ltd, Beijing, China )was then administered at a rate of $200 \mathrm{~mL} / \mathrm{hr}$ until LOC. At that time, the infusion rate was adjusted according to the patient's weight (eg, for patients weighing $70 \mathrm{~kg}$, the rate was $70 \mathrm{~mL} / \mathrm{hr}$ ) until laryngeal mask airway (LMA) insertion was complete. Based on the Minto model, we targeted the effect-site concentration of remifentanil at $1.5 \mathrm{ng} / \mathrm{mL}$. The concentration of remifentanil was diluted to $20 \mu \mathrm{g} / \mathrm{mL}$. After the corneal reflex test, rocuronium $0.6 \mathrm{mg} / \mathrm{kg}$ was administered to facilitate insertion of LMA, allowing subsequent control of ventilation. The BIS sensor (BISTM XP) was applied according to the manufacturer's guidelines with a smooth rate at $30 \mathrm{~s}$. The investigator was blinded to the study groups and required patients to open their eyes repeatedly every $10-20 \mathrm{~s}$ during induction. We defined LOC as the loss of reaction to auditory commands. Following the patient's LOC, rocuronium may generate movement which is presumed to be secondary to pain at the site of injection. ${ }^{8}$ Visible arm movement during rocuronium administration was defined as rocuronium injection pain.

Baseline HR and mean arterial pressure (MAP) were measured before induction (T0). During induction, the hemodynamic profiles were monitored at the following timepoints: LOC (T1), administration of rocuronium (T2), insertion of LMA (T3), and 1 min after LMA insertion (T4). At LOC, BIS, presence of spontaneous breathing, and corneal reflex were noted. MAP was monitored at an interval of $1 \mathrm{~min}$ during anesthesia induction and an interval of 5 mins during maintenance. Corneal reflex was tested by dripping 2-3 drops of $0.9 \%$ normal saline.

After confirmation of appropriate LMA placement, etomidate or propofol was discontinued. Sevoflurane and remifentanil were continued to maintain anesthesia. The infusion rate of remifentanil was adjusted according to surgical stimuli. Patients were provided a bolus of morphine at the end of surgery and were visited the next day to determine awareness of the corneal reflex test.

In our pilot study, which included 12 patients in each group, we found that the MAP at LMA insertion was 72.8 (10.3) $\mathrm{mmHg}$ in the etomidate group and 65.3 (12.7) $\mathrm{mmHg}$ in the propofol group. A sample size of 39 patients or more for each group would be needed to detect statistically significant differences between groups when applying power of 0.8 with level 0.05 . Hemodynamic changes were the primary focus, with all other measurements viewed as secondary outcomes.

We report data as mean $\pm \mathrm{SD}$, unless otherwise noted. Unpaired Student's $t$-test was used to analyze parametric data, and $X^{2}$ or Fisher's exact test was applied to examine nominal data. Two-way ANOVA with repeated measurements and interaction between time and group factors was used to evaluate differences in hemodynamic profiles between groups. Differences in hemodynamic variables between groups at different time points were compared by post hoc Bonferroni test. Significance was set at $p$-values $<0.05$. 


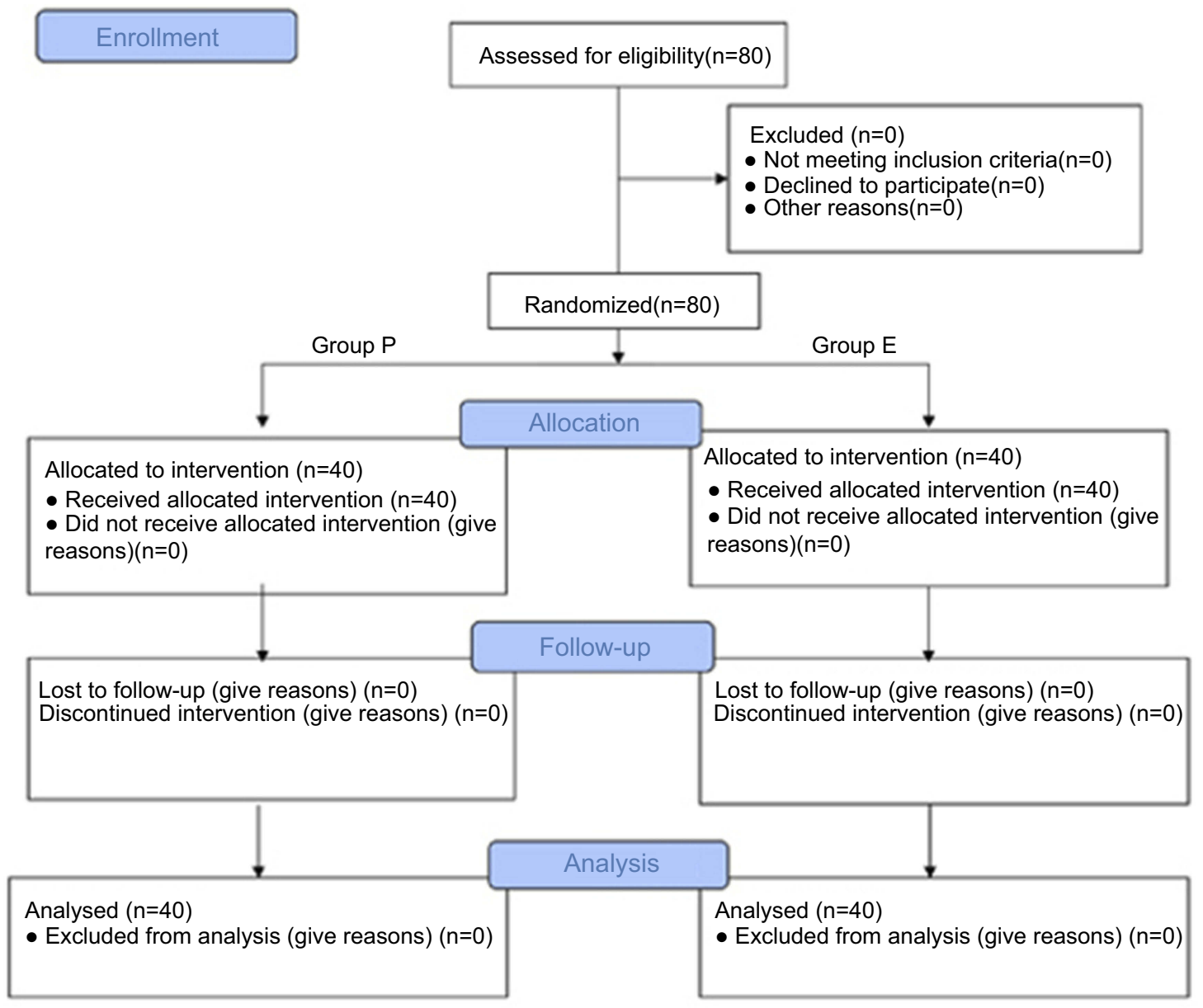

Figure I CONSORT flow diagram. Eighty patients were randomized.

Abbreviations: $P$, propofol group; $\mathrm{E}$, etomidate group.

\section{Results}

Eighty patients enrolled into and completed the study [Group E $(n=40)$, Group P $(n=40)$ ] (Figure 1). Patient characteristics were examined (Table 1). Gender, age, weight, height, and baseline BIS were not significantly different between groups.

HRs in Group E decreased from $T_{0}$ to $T_{1}$ and returned to baseline at $T_{2}, T_{3}$, and $T_{4}$. MAPs in Group E decreased

Table I Demographic characteristics

\begin{tabular}{|l|l|l|l|}
\hline Parameter & $\begin{array}{l}\text { Group E } \\
(\mathbf{n = 4 0 )}\end{array}$ & $\begin{array}{l}\text { Group } \boldsymbol{P} \\
(\mathbf{n = 4 0 )}\end{array}$ & $\boldsymbol{P}$ \\
\hline Sex (female/male) & $23 / 17$ & $21 / 19$ & 0.8 \\
Age (years) & $41.73 \pm 10.17$ & $40.28 \pm 13.11$ & 0.6 \\
Weight (kg) & $62.63 \pm 8.83$ & $63.95 \pm 9.28$ & 0.5 \\
Height & $165.08 \pm 6.83$ & $165.1 \pm 7.52$ & 1 \\
BIS value & $96.5 \pm 1.7$ & $96.9 \pm 1.0$ & 1 \\
\hline
\end{tabular}

Notes: Data are expressed as mean (SD) or number of patients. Abbreviations: BIS, bispectral index; E, etomidate group; P, propofol group. from $T_{0}$ to $T_{1}$ and returned to baseline at $T_{2}$. HRs and MAPs in Group $P$ decreased from $T_{0}$ to $T_{4}$. Two-way ANOVA with repeated measurement analysis showed there was no statistically significant interaction of time and group based on HR between the two groups, while a post hoc Bonferroni test showed that HRs at $T_{2}, T_{3}$, and $\mathrm{T}_{4}$ were significantly higher in Group $\mathrm{E}$ than those in Group $\mathrm{P}$ (Figure 2A, $P=0.1$ ). Repeated-measurement ANOVA with a post hoc Bonferroni test showed a difference in MAP over times between the two groups, with MAPs at $T_{3}$ and $T_{4}$ being significantly higher in Group E than those in Group P (Figure 2B, $P=0.003$ ).

The mean time to LOC was significantly shorter with etomidate than with propofol $(129.5 \mathrm{~s}$ vs $189.5 \mathrm{~s}$, $P<0.0001)$. The average dose to reach LOC was $0.23 \mathrm{mg} / \mathrm{kg}$ for etomidate and $1.64 \mathrm{mg} / \mathrm{kg}$ for propofol. At LOC, BIS was significantly lower in Group E compared with Group P (46.3 vs $52.9, P=0.01)$. Thirtytwo patients $(80 \%)$ were spontaneously breathing at LOC 
A

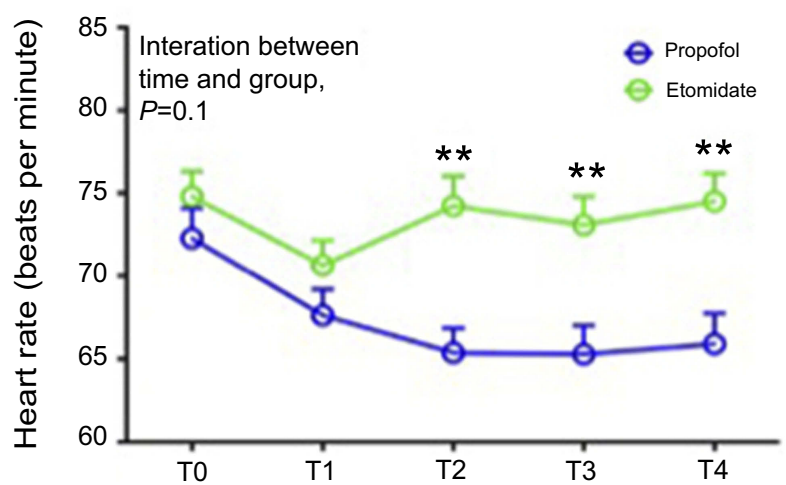

B

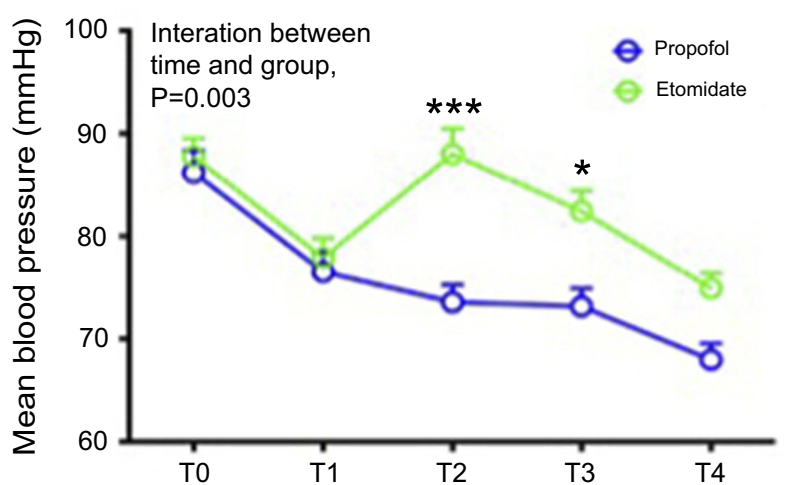

Figure 2 Changes in heart rate, HR (Panel A) and mean arterial pressure, MAP (Panel B) at the indicated time points during induction in the propofol $(P)$ and etomidate (E) groups. $T_{0}$, baseline value before anesthesia; $T_{1}$, at loss of consciousness; $T_{2}$, at administration of rocuronium; $T_{3}$, insertion of laryngeal mask airway; $T_{4}$, I min after $L M A$ insertion. $* P<0.05, * * P<0.01$, $* * * P<0.001$; repeated-measurement ANOVA.

in Group E, whereas only seven patients (17.5\%) were breathing at LOC in Group P, $P<0.0001$. Similarly, corneal reflex was maintained in a significantly greater number of Group E patients compared with those in Group P (34 patients vs 4 patients, $P<0.0001)$. More patients in Group $\mathrm{E}$ than in Group $\mathrm{P}$ presented with pain from rocuronium injection, but the difference was not significant (12 patients vs 8 patients, $P=0.4$ ). The incidence of etomidateinduced myoclonus was $17.5 \%$ (Table 2).

No patients reported awareness of the corneal reflex test.

\section{Discussion}

We compared etomidate vs propofol infusion in terms of hemodynamic changes, existence of spontaneous breathing, and corneal reflex during the induction of anesthesia.

Table 2 Time to LOC, agent dosage, BIS value, respiratory variables, and adverse events

\begin{tabular}{|c|c|c|c|}
\hline & $\begin{array}{l}\text { Group E } \\
(n=40)\end{array}$ & $\begin{array}{l}\text { Group } P \\
(n=40)\end{array}$ & $p$ \\
\hline Dosage (mg.kg $\left.{ }^{-1}\right)$ & $0.23 \pm 0.05$ & $1.64 \pm 0.34$ & \\
\hline Time to LOC (s) & $129.5 \pm 25.8$ & $189.5 \pm 52.1$ & $<0.0001$ \\
\hline BIS value at $L O C$ & $46.3 \pm 14.3$ & $52.9 \pm 8.6$ & 0.01 \\
\hline $\begin{array}{l}\text { Presence of spontaneous } \\
\text { breathing }\end{array}$ & $32(80 \%)$ & 7 (I7.5\%) & $<0.0001$ \\
\hline Presenceof corneal reflex & 34 (85\%) & $4(10 \%)$ & $<0.0001$ \\
\hline Rocuronium injection pain & $12(30 \%)$ & $8(20 \%)$ & 0.4 \\
\hline $\begin{array}{l}\text { Etomidate-induced } \\
\text { myoclonus }\end{array}$ & 7 (I7.5\%) & & \\
\hline
\end{tabular}

Notes: Data are expressed as number (percentage) or mean (SD).

Abbreviations: LOC, loss of consciousness; BIS, bispectral index; E, etomidate group; P, propofol group.
Etomidate provided a more stable hemodynamic profile during induction. Among patients in Group E, at LOC, the BIS value was lower, and more patients exhibited spontaneous breathing and corneal reflexes.

In our study, etomidate exhibited fewer hemodynamic changes when compared with propofol. MAP and HR decreased by approximately $10 \%$ at LOC when compared with baseline in both groups. At other time points, MAP and HR were higher in the etomidate group. The effect of etomidate on hemodynamic stability may be caused by its lack of effect on baroreceptor function and the sympathetic nervous system. ${ }^{3,4,9}$ The increase of HR and MAP from T1 to $\mathrm{T} 2$ in Group $\mathrm{E}$ indicated that injection pain of rocuronium more profoundly activated the sympathetic nervous system. However, Goodchild et al found that propofol did not cause a negative inotropic effect or relax arteries at clinical concentrations. ${ }^{5}$

Anesthetics cause central nervous system depression. In 1985, Thornton et al reported that etomidate suppresses cortical activity in a dose-dependent manner but does not affect brainstem response. ${ }^{10}$ This finding indicates that anesthetic responses result from changes at the cortical level. Thus, effects of anesthetics, such as reduced blood pressure and apnea, may be indirectly related to the brainstem. By contrast, Tooley et al found that propofol alters the latency of brainstem wave $\mathrm{V}$, indicating that propofol suppresses brainstem response. ${ }^{11}$ Even at the same depth of anesthesia guided by the BIS value, Möller et al reported that etomidate caused fewer hemodynamic changes than propofol during induction. ${ }^{7}$ Furthermore, even with a lower BIS value at LOC, more patients in Group E maintained spontaneous breathing and corneal 
reflex. These findings demonstrate that etomidate has fewer effects on brainstem function.

Propofol is the agent most commonly used for induction of anesthesia. The recommended bolus dose is $2.5 \mathrm{mg} / \mathrm{kg}$ to achieve LOC in $95 \%$ of subjects. However, this dose may induce a greater degree of hypotension relative to opioids. ${ }^{12}$ Decreasing the rate of propofol administration may prevent hypotension before tracheal intubation. A lower rate of propofol infusion can also reduce the total propofol dose required for LOC. ${ }^{13}$ Etomidate is a candidate agent for induction of anesthesia. ${ }^{13}$ An outstanding property of etomidate is maintenance of hemodynamic stability compared with effects of propofol. ${ }^{2,7,13-15}$ In our study, we delivered both agents by infusion. The average dose of propofol to reach LOC was $1.64 \mathrm{mg} / \mathrm{kg}$ and that of etomidate was $0.23 \mathrm{mg} / \mathrm{kg}$.

BIS is closely related to the depth of anesthesia and LOC for propofol and etomidate. ${ }^{16-18}$ In our study, we defined LOC as loss of response to verbal command. The BIS value was 46.3 in Group E and 53.4 in Group P at LOC. These values are within the recommended BIS value range of 40 to 60 for welltolerated anesthesia. ${ }^{18}$ Furthermore, no patients recalled the corneal test. In our study, when the two agents were delivered by infusion, the mean time to LOC was $129.5 \mathrm{~s}$ for etomidate and $189.5 \mathrm{~s}$ for propofol. These results are in contrast to those reported by Möller et al, ${ }^{7}$ who found that propofol exerted actions, such as time to palpebral reflex loss and BIS 60, more rapidly than etomidate. Differences in drug infusion strategy may contribute to the different findings.

Myoclonus is a common adverse effect of etomidate, with an incidence of approximately $70 \% .{ }^{19}$ In our study, the incidence of myoclonus was $17.5 \%$. Slow delivery ${ }^{2}$ and opioids ${ }^{20}$ can decrease the incidence of myoclonus. Another well-known side effect of etomidate is adrenocortical suppression. However, even vulnerable patients do not experience adrenal crisis in response to etomidate. ${ }^{2}$ A systematic review and metaanalysis revealed that in patients with sepsis a single dose of etomidate did not increase mortality. ${ }^{21}$

There were several limitations in our study. First, the patients in our study were relatively healthy and did not harbor hemodynamic vulnerabilities as found among the elderly. However, our study confirmed that etomidate maintained hemodynamic stability to a greater extent than that achieved by propofol. Second, etomidate and propofol infusion doses were not calculated by equipotency. We manually delivered both agents at a constant infusion rate until LOC. Recently some authors used the closed-loop anesthesia by propofol and calculated the percentage of time in which the BIS was in the range. ${ }^{22}$ Future studies regarding manual vs closed-loop administration and percentage of time in which the BIS was in a specified value should be carried out. Third, except for corneal reflex, brainstem function was not specifically evaluated. Further study is warranted to test the hypothesis that etomidate has fewer effects on brainstem function compared with propofol.

\section{Conclusion}

In summary, etomidate causes fewer hemodynamic changes than propofol during anesthesia induction. At LOC, the BIS value was lower in Group E. Spontaneous breathing and corneal reflex were preserved in more patients who received etomidate compared with those who received propofol. These findings suggest that etomidate may have fewer effects on brainstem function.

\section{Availability of data and materials}

The datasets used or analyzed during the current study are available from the corresponding author upon reasonable request.

\section{Ethical Standards}

The authors assert that all procedures contributing to this work comply with the ethical standards of the relevant national and institutional guidelines on human experimentation and with the Helsinki Declaration of 1975, as revised in 2008.

\section{Acknowledgments}

The authors thank the anesthesiologists, surgeons, and anesthesia assistants who participated in this study.

\section{Author contributions}

All authors contributed to data analysis, drafting or revising the article, gave final approval of the version to be published, and agree to be accountable for all aspects of the work.

\section{Disclosure}

The authors report no conflicts of interest in this work.

\section{References}

1. Bendel S, Ruokonen E, Pölönen P, Uusaro A. Propofol causes more hypotension than etomidate in patients with severe aortic stenosis: a double-blind, randomized study comparing propofol and etomidate. Acta Anaesthesiol Scand. 2007;51:284-289. 
2. Song JC, Lu ZJ, Jiao YF, et al. Etomidate anesthesia during ERCP caused more stable hemodynamic responses compared with propofol: a randomized clinical trial. Int $J$ Med Sci. 2015;12:559-565. doi:10.7150/ijms. 11521

3. Ebert TJ, Muzi M, Berens R, et al. Sympathetic responses to induction of anesthesia in humans with propofol or etomidate. Anesthesiology. 1992;76:725-733.

4. Bentley GN, Gent JP, Goodchild CS. Vascular effects of propofol: smoothmuscle relaxation in isolated veins and arteries. J Pharm Pharmacol. 1989;41:797-798.

5. Goodchild CS, Serrao JM. Cardiovascular effects of propofol in the anaesthetized dog. Br J Anaesth. 1989;63:87-92. doi:10.1093/bja/ 63.1 .87

6. Goodchild CS, Serrao JM. Propofol induced cardiovascular depression: science and art. Br J Anaesth. 2015;115:641-642. doi:10.1093/ bja/aev320

7. Möller Petrun A, Kamenik M. Bispectral index-guided induction of general anesthesia in patients undergoing major abdominal surgery using propofol or etomidate: a double-blind, randomized, clinical trial. Br J Anaesth. 2013;110:388-396. doi:10.1093/bja/aes416

8. Shevchenko Y, Jocson JC, McRae VA, et al. The use of lidocaine for preventing the withdrawal associated with the injection of rocuronium in children and adolescents. Anesth Analg. 1999;88:746-748.

9. Sprung J, Ogletree-Hughes ML, Moravec CS. The effects of etomidate on the contractility of failing and nonfailing human heart muscle. Anesth Analg. 2000;91:68-75.

10. Thornton C, Heneghan CPH, Navaratnarjah M, et al. Effect of etomidate on the auditory evoked response in man. Br J Anaesth. 1985;57:554-561. doi:10.1093/bja/57.6.554

11. Tooley MA, Greeenslade GL, Prys-Roberts C. concentration related effects of propofol on the auditory evoked response. Br J Anaesth. 1996;77:720-726. doi:10.1093/bja/77.6.720

12. Billard V, Moulla F, Bourgain JL, et al. hemodynamic response to induction and intubation propofol/fentanyl interaction. Anesthesiology. 1994;81:1384-1393.
13. Peacock JE, Lewis RP, Reilly CS, Nimmo WS. Effect of different rates of infusion of propofol for induction of anesthesia in elderly patients. Br J Anaesth. 1990;65:346-352. doi:10.1093/bja/65.3.346

14. Davis PJ, Cook DR. Clinical pharmacokinetics of the newer intravenous anaesthetic agents. Clin Pharmacokinet. 1986;11:18-35. doi:10.2165/00003088-198611010-00002

15. Reich DL, Hossain S, Krol M, et al. Predictors of hypotension after induction of general anesthesia. Anesth Analg. 2005;101:622-628. doi:10.1213/01.ANE.0000175214.38450.91

16. Kaneda K, Yamashita S, Woo S, et al. Population pharmacokinetics and pharmacodynamics of brief etomidate infusion in healthy volunteers. J Clin Pharm. 2011;51:482-491. doi:10.1177/009127001 0369242

17. Liu J, Singh H, White PF. Electroencephalographic bispectral index correlates with intraoperative recall and depth of propofol-induced sedation. Anesth Analg. 1997;84:185-189.

18. Glaas PS, Bloom M, Kearse L, et al. Bispectral analysis measures sedation and memory. Effects of propofol, midazolam, isoflurane, and alfentanil in healthy volunteers. Anesthesiology. 1997;86: 836-847.

19. Schneider G, Gelb AW, Schmeller B, et al. Detection of awareness in surgical patients with EEG-based indices: bispectral index and patient state index. Br J Anaesth. 2003;91:329-335. doi:10.1093/ bja/aeg188

20. Kelsaka E, Karakaya D, Sarihasan B, et al. Remifentanil pretreatment reduces myoclonus after etomidate. J Clin Anesth. 2008;18:83-86. doi:10.1016/j.jclinane.2005.05.004

21. Miner JR, Danahy M, Moch A, Biros M. Randomized clinical trial of etomidate versus propofol for procedural sedation in the emergency department. Ann Emerg Med. 2007;49:15-22. doi:10.1016/j. annemergmed.2006.06.042

22. Cotoia A, Mirabella L, Beck R, et al. Effects of closed-loop intravenous anesthesia guided by the Bispectral index in adult patients on emergence delirium. A randomized controlled study. Minerva Anestesiol. 2018;84:437-446. doi:10.23736/S0375-9393.17.11915-2

\section{Publish your work in this journal}

Drug Design, Development and Therapy is an international, peerreviewed open-access journal that spans the spectrum of drug design and development through to clinical applications. Clinical outcomes, patient safety, and programs for the development and effective, safe, and sustained use of medicines are a feature of the journal, which has also been accepted for indexing on PubMed Central. The manuscript management system is completely online and includes a very quick and fair peer-review system, which is all easy to use. Visit http://www. dovepress.com/testimonials.php to read real quotes from published authors. 